\title{
Differences in Regional Distribution and Inequality in Health-Resource Allocation on Institutions, Beds, and Workforce: A Longitudinal Study in Shanghai, China
}

\section{Enhong Dong}

Shanghai University of Medicine and Health Sciences https://orcid.org/0000-0002-7754-5143

\section{Xiaoting Sun}

Tongji University School of Medicine

Lufa Zhang

Shanghai Jiao Tong University

\section{Minjie Chen}

Shanghai Jiao Tong University School of Medicine Affiliated Renji Hospital

Hongmei Wang

University of Nebraska Medical Center

\section{Ting Xu}

Shanghai University of Medicine and Health Sciences

Tao Wang ( $\sim$ happywt0403@sina.com )

College of Arts and Media, Tongji University

\section{Research}

Keywords: Health-care resources, regional difference, inequality, central districts; suburban districts

Posted Date: August 21st, 2020

DOI: https://doi.org/10.21203/rs.3.rs-62172/v1

License: (c) (i) This work is licensed under a Creative Commons Attribution 4.0 International License. Read Full License 


\section{Title page}

\section{Differences in regional distribution and inequality in health-resource allocation on}

\section{institutions, beds, and workforce: A longitudinal study in Shanghai, China}

Enhong Dong ${ }^{12}$, Xiaoting Sun $^{34}$, Lufa Zhang ${ }^{5}$, Minjie Chen ${ }^{6}$, Hongmei Wang ${ }^{7}$,Ting Xu, ${ }^{1}$ Tao Wang ${ }^{48^{*}}$,

${ }^{1}$ School of Nursing and Health Management, Shanghai University of medicine \& Health Science, 279

Zhouzhu Road, Pudong new District, Shanghai 201318, Shanghai, China; ${ }^{2}$ School of Media and

Communication, Shanghai Jiao Tong University, Shanghai 200240, Shanghai, China; ${ }^{3}$ Shanghai Tenth

People's Hospital affiliated to Tongji University, Shanghai, China; ${ }^{4}$ Tongji University School of Medicine,

Shanghai, China; ${ }^{5}$ School of International and Public Affairs, Shanghai Jiao Tong University.1954

Huashan Road, Xuhui District, Shanghai 200030, Shanghai, China; ${ }^{6}$ Department of Outpatient and

Emergency, Renji Hospital Affiliated to Shanghai Jiao Tong University School of Medicine, NO.160

Pujian Road, Pudong new District, Shanghai,200127,China; ${ }^{7}$ Department of Health Services Research \& Administration, College of Public Health, 984350 University Nebraska Medical Center, Omaha, NE 68198-4350; ${ }^{8}$ College of Arts and Media, Tongji University, Shanghai 200092 , China.

*Corresponding author: E-mail: Tao Wang happywt0403@sina.com

E-HD ,X-TS and L-FZ contributed equally. 


\begin{abstract}
Background: The distribution of health-care resources is a critical component of health-care access, and equity is a basic principle of health-resource allocation, and foundational to achieving fairness in the provision of health services. China and its local Shanghai's government has implemented measures to allocate health-care resources with the equity as one of the major goals since 2009. The aim of this study was to analyze differences in regional distribution and inequality in health-resource allocation on institutions, beds, and workforce in Shanghai over 7 years.
\end{abstract}

Methods: A longitudinal survey using 2010-2016 data, which were collected for analysis. The study was conducted health-resource allocation on institutions, beds, and workforce in Shanghai, China. Five health-resource indicators were used to measure health-resource distribution at the city and district levels. Furthermore, the Theil index was calculated to measure inequality of health-resource allocation.

Results: All quantities of health-care resources per 1000 people increased across Shanghai districts from 2010 to 2016. Compared with suburban districts, the central districts had higher ratios on five health-care resource indicators, and faster average growth in the bed and nurse indicator, and slower growth in the institution, technician and doctor indicator. The Theil indices of the technicians, doctors, nurses and beds had higher values than that of institutions every year from 2010 to 2016; furthermore, the Theil indices of the indicators, except for doctors in hospitals, all exhibited downward time trends.

Conclusions: Increased health-care resources and reduced inequality of health-resource allocation in Shanghai during the 7 years indicated that the measures taken by the Shanghai government in the new round of healthcare reform in China since 2009 had been successful. Meanwhile there still existed regional difference between urban and rural areas and inequality between institution and workforce, especially doctors.

Keywords: Health-care resources, regional difference, inequality, central districts; suburban districts 


\section{Background}

Reasonable health-resource allocation is essential to achieving health service equity, which contributes to public health outcomes and mitigates social conflict[1-3]. In many countries, healthcare reform aims to provide universal and equitable access to health care, which is recognized as a fundamental human right. The distribution of health-care resources is a critical component of health-care access. Furthermore, equity is a basic principle of health-resource allocation, and it is foundational to achieving fairness in the provision of health services. Many studies have demonstrated that highly accessible health care can play a crucial role in promoting regional health equity. The equitable allocation of health-care resources helps deliver health-care resources to those most in need and ensures accessibility to basic health services as well as fairness for vulnerable populations[4]. Moreover, inequality in health-care resources has adverse consequences, such as the uneven distribution of health-care allocation, which in turn leads to growing inequalities between the rich and poor with respect to health and the economic burden of disease[5]. In 2009, China launched a new round of healthcare reform with the aim of providing households with secure, efficient, convenient, equitable, and affordable health-care services by reversing the market-oriented health system into one with universal benefits. The reform strengthened the government's role in healthcare, its commitment to equity, and its willingness to experiment with regulated market approaches. Besides genetic characteristics, the Chinese healthcare system also has some more special features. Take the health financing system as an example, it collects revenues from three main sources: government expenditure, social expenditure and out-of-pocket (OOP) payments in the domestic classification. The revenues are distributed through the basic medical security system consisting of Basic Medical Insurance (BMI) schemes and Medical Financial Assistance (MFA) schemes for the poor to cover urban and rural residents in China. Under BMI, more specifically, employees in urban areas are covered by Urban Employee Basic Medical Insurance (UEBMI), unemployed residents in urban areas are covered by Urban Residents Basic Medical Insurance (URBMI) and residents in rural areas are covered by New Rural Cooperative Medical System (NRCMS). The MFA is the security net for the poor in both urban and rural areas, which helps them to enroll in basic medical insurance and also provides extra reimbursement for medical expenses. The public 
health system, which is mainly financed by the government, provides basic public health services to all residents free of charge. Accordingly, since 2009, the Shanghai government has implemented corresponding measures to allocate health-care resources between central districts and rural ones, conforming tightly to the national health reform strategies and guidelines and three tenets for Shanghai's healthcare reform (build a foundation, manage for the long term, and make reforms sustainable). For example, in 2009, shanghai government spent approximately $\$ 1$ billion to carry out a policy named " $5+3+1$ " to strengthen the role of public hospitals, and to deepen health-care reforms. Under the policy, nine tertiary hospitals in rural areas were constructed and a " 1560 " accessible radius to health care were formulated, so that patients in urban areas can walk to a nearest medical institution within 15 minutes, and patients in suburban districts can visit a tertiary hospital within 60 minutes by public transportation[6]. As a result of these measures, nine new tertiary hospitals were built and 6000 beds were provided in the outer rings of Shanghai. Rural patients no longer had to drive an hour or two to visit tertiary hospitals in the city center. With the increase number of institutions, beds and workforce transferring from "mother hospitals" to new hospitals, the distribution of health-care resources has become more balanced $[7,8]$. However, many studies examined variations in the quantity and inequality in

health-resource allocation in China have noted widening urban-rural disparities in health-care resources across China[9-14], including the one conducted in Shanghai[15]. However, they have overlooked the difference over time in health-resource allocation as well as its association with China's 2009 healthcare reform. Considering the overall goal of China's new health-care guidelines and plans to promote more equitable and efficient health-care resource distribution, it is essential to study the differences in health-resource distribution and the inequity of allocation in Shanghai over time since the 2009 reform.

Therefore, the purpose of this study was first, to investigate regional difference in healthresource distribution and second, to describe the inequity of their allocation over 7 years (20102016) in Shanghai, in order to see if both of them have changed after the new round of health reform in China since 2009.

\section{Methods}

\section{Patient and public involvement}

This study used secondary data from Yearbooks (2010-2016) in Shanghai of China and did not require patient or public involvement. 


\section{Data source}

This study used data from the Shanghai Medical Statistical Yearbook from 2010-2016 and the Shanghai Statistical Yearbook from 2010-2016, which were published by the Shanghai Health Commission and Shanghai Statistics Bureau, respectively. The indicators used in the study included the number of health institutions, the number of beds, the number of beds, the number of technicians, the number of doctors, and the number of nurses. These data were taken from the 2010 to 2016 editions of the Shanghai Medical Statistical Yearbook. Table 1 presents all 5 indicators and their definitions along with how they were measured. Per capita measures of all the indicators were calculated after obtaining the annual population of the whole city and every administrative district from the 2010-2016 editions of the Shanghai Statistical Yearbook.

Table 1 Indicators of health-resource allocation, their definitions, and how they were measured

\begin{tabular}{|c|c|c|}
\hline Indicator & Definition & $\begin{array}{l}\text { How indicator was } \\
\text { measured }\end{array}$ \\
\hline $\begin{array}{l}\text { Number of } \\
\text { institutions }\end{array}$ & $\begin{array}{l}\text { It refers to the institutions such as hospitals, primary health care } \\
\text { institutions, professional public health agencies and other } \\
\text { healthcare institutions which have obtained the legal registration } \\
\text { certificates from the health administrative departments in China }\end{array}$ & $\begin{array}{l}\text { Number of institutions } \\
\text { divided by the } \\
\text { population }\end{array}$ \\
\hline Number of beds & $\begin{array}{l}\text { It refers to the actual number of beds in medical institutions, } \\
\text { including formal beds, simple beds, care beds, beds are being } \\
\text { disinfected or repaired, not including neonatal beds, pre- } \\
\text { delivery beds, observation beds, temporary beds and the } \\
\text { accompany beds for patients' family }\end{array}$ & $\begin{array}{l}\text { Number of beds } \\
\text { divided by the } \\
\text { population }\end{array}$ \\
\hline $\begin{array}{l}\text { Number of } \\
\text { technicians }\end{array}$ & $\begin{array}{l}\text { It refers to the workforce who assist medical staff complete } \\
\text { tasks around their assigned unit or clinic and accommodate } \\
\text { patient needs, including pharmacists and radiologists; registered } \\
\text { nurses were excluded }\end{array}$ & $\begin{array}{l}\text { Number of technicians } \\
\text { in hospitals divided by } \\
\text { the population }\end{array}$ \\
\hline $\begin{array}{l}\text { Number of } \\
\text { doctors }\end{array}$ & $\begin{array}{l}\text { It refers to the physicians who hold a practicing physician } \\
\text { certificate, including practicing physicians and assistants in } \\
\text { China. Those who are engaged in the management of health } \\
\text { workers as part of the health workforce, such as presidents, vice } \\
\text { presidents, and party secretaries were excluded }\end{array}$ & $\begin{array}{l}\text { Number of doctors } \\
\text { divided by the } \\
\text { population }\end{array}$ \\
\hline $\begin{array}{l}\text { Number of } \\
\text { nurses }\end{array}$ & $\begin{array}{l}\text { It refers to registered nurses who have obtained the legal } \\
\text { practicing nurses' certificates. Those who engaged in the } \\
\text { management of health workers are not included as health } \\
\text { workforce, such as president, vice president, party secretary }\end{array}$ & $\begin{array}{l}\text { Number of nurses } \\
\text { divided by the } \\
\text { population }\end{array}$ \\
\hline
\end{tabular}

Shanghai is one of four directly controlled municipalities of the People's Republic of China, and it is further divided into 16 districts, including seven urban and nine suburban districts. Shanghai's urban administrative divisions are as follows: Huangpu, Xuhui, Changning, Jing'an, Putuo, Hongkou, and Yangpu. Its rural administrative divisions are Minhang, Baoshan, Jiading, Pudong New Area, Jinshan, Songjiang, Qingpu, Fengxian, and Chongming. Over 7 years from 
2010 to 2016, Shanghai had three administration division mergers aimed at facilitating the longterm development of all the districts involved, enhancing the administrative efficiency of urban function and resource distribution for the city, as well as reducing administrative costs. Specifically, in 2011, Luwan District was merged with a neighboring district to form the new Huangpu District. Zhabei District was merged with Jing'an District in 2015; and Chongming County was upgraded to Chongming District in 2016. To maintain data comparability, we formatted the new data of the 16 administration divisions by integrating the data of the two merged districts of Luwan and Zhabei into those of Huangpu and Jing'an, respectively.

\section{Data analysis}

The annual growth rates (AGRs) of the five types of healthcare resource were also calculated from 2010 to 2016. The formula of AGR is as follows:

$$
\mathrm{AGR}=\sqrt[n]{\frac{B}{A}}-1
$$

where B is the quantity of the five types of healthcare resource in 2016, A is the quantity of the five types of healthcare resource in 2010, and n represents the number of years. We used AGR (Average Growth Rate) instead of GR (Growth Rate) to measure the time trends of healthcare resources for the advantages of its accuracy to calculate historical tracks and comparability of the relative performance of the health-care resource allocation.

Many measures exist for evaluating the equity of health-resource allocation, such as the Lorenz curve, Gini coefficient, and Theil index. The Theil index is a statistic primarily used to measure income inequality or other economic phenomena among different individuals or within varied groups. It is a special case of the generalized entropy index and one of the most widely used measures of inequality in regional economic development. The Theil index was proposed by econometrician Henri Theil at Erasmus University Rotterdam [16], and it can be formulated as follows:

$$
T=\frac{1}{n} \sum_{i=1}^{n} \frac{y_{i}}{\bar{y}} \log \left(\frac{y_{i}}{\bar{y}}\right),
$$

where $T$ is the Theil index, which represents income allocation inequality, and $y_{i}$ and $\bar{y}_{\text {is the }}$ income of individual $i$ and the average income of the population, respectively. 
The Theil index has another form to measure the inequality between different groups, which is known as the between-region difference. This formula can be written as follows:

$$
T=\sum_{i=1}^{k} w_{i} \ln \left(\frac{w_{i}}{e_{i}}\right),
$$

where $w_{i}$ represents the proportion of the income of group $i$ accounting for the total income of all groups and ${ }^{e_{i}}$ represents the proportion of the people in group $i$ accounting for the overall population of all groups. In this study, we defined $w_{i}$ as the proportion of health-care resources in district $i$ accounting for the resources of the whole city, and we defined $e_{i}$ as the proportion of the people in district $i$ accounting for the overall population of the city. The value of the Theil index ranges from 0 to 1 and 0 represents perfect equality, while 1 means completely unequal. Statistical analyses were performed using Stata statistical software version 15.0 (StataCorp LP, College Station, TX, U.S.)[17], and maps were generated using ArcGis 10.6 (Environmental Systems Research Institute, Redlands, CA, USA)[18].

\section{Results}

\section{Differences in regional distribution of health-resource allocation at city and district levels in Shanghai from 2010 to 2016}

Table 2 presents descriptive statistics of indicators of health-resource allocation in Shanghai. Table 3 presents changes in numbers and AGRs related to health-resource allocation in Shanghai. Further details of changes for each indicator with whiskers box plot per every year from 2010 to 2016 are presented in appendix (see the additional file 1). As indicated by the table, the healthcare resources increased gradually from 2010 to 2016, the quantities of health-care resources per 1000 of the population all increased, the ratios of health institutions, beds, technicians, doctors and nurses per 1000 population rose by $6.99 \%, 2.67 \%, 3.81 \%, 3.30 \%$ and $5.13 \%$ annually from 2010 to 2016, respectively. The number of institutions grew faster than the health workforce did overall, and the number of beds per 1000 people grew the slowest among the five types of indicators. For example, from 2010 to 2016, the number of institutions per 1000 people increased by $6.99 \%$ annually, the numbers of technicians, doctors and nurses per 1000 people annually increased by $3.81 \%, 3.30 \%$ and $5.13 \%$, respectively, while the number of beds rose by $2.67 \%$, only accounting for $3 / 8$ of AGR of the institution indicators during the same period (Table 3). 
Table 2 Descriptive Statistics of indicators of health-resource allocation in Shanghai (20102016; per 1000)

\begin{tabular}{lrrrrr}
\hline Indicators & Obs. & Min. & Max. & Mean. & Median \\
Number of institutions & 112 & 0.07 & 0.51 & 0.21 & 0.17 \\
Number of beds & 112 & 2.05 & 17.52 & 5.98 & 4.59 \\
Number of technicians & 112 & 3.08 & 28.08 & 8.20 & 5.63 \\
Number of doctors & 112 & 1.23 & 10.03 & 3.02 & 2.08 \\
Number of nurses & 112 & 1.16 & 12.92 & 3.58 & 2.26 \\
\hline
\end{tabular}

Table 3 Changes in the numbers and AGRs related to health-resource allocation in Shanghai (2010-2016; per 1000)

\begin{tabular}{lcccccccc}
\hline \multicolumn{1}{c}{ Indicator } & $\mathbf{2 0 1 0}$ & $\mathbf{2 0 1 1}$ & $\mathbf{2 0 1 2}$ & $\mathbf{2 0 1 3}$ & $\mathbf{2 0 1 4}$ & $\mathbf{2 0 1 5}$ & $\mathbf{2 0 1 6}$ & AGR \\
\hline Number of institutions & 0.14 & 0.14 & 0.15 & 0.20 & 0.21 & 0.21 & 0.21 & $6.99 \%$ \\
Number of beds & 4.56 & 4.56 & 4.60 & 4.73 & 4.84 & 5.08 & 5.34 & $2.67 \%$ \\
Number of technicians & 5.88 & 5.92 & 6.14 & 6.47 & 6.76 & 7.05 & 7.36 & $3.81 \%$ \\
Number of doctors & 2.23 & 2.22 & 2.28 & 2.40 & 2.53 & 2.61 & 2.71 & $3.30 \%$ \\
Number of nurses & 2.43 & 2.51 & 2.66 & 2.81 & 2.97 & 3.12 & 3.28 & $5.13 \%$ \\
\hline
\end{tabular}

AGR = Average Growth Rate.

Fig.1 showed the time trends and geographic distribution of the health-care resource allocation across all Shanghai's districts from 2010 to 2016 (for space constraints, only data from 2010, 2013 and 2016 were presented in Fig. 1). As for administrative divisions, from 2010 to 2016, an overall increasing trend was observed in the numbers of institutions, beds and workforce per 1000 people across all districts, meanwhile with central districts with darker red indicating having the highest health-care resource densities than suburban districts with darker blue indicating the lowest density. This indicated an unchanged distribution concentration both in health-care facility and workforce resource allocation among central areas other than rural ones in Shanghai. However, among the different types of health-care resource, central and suburban districts grew differently during the same period. Compared with suburban districts, central districts grew faster in the AGRs of the beds and nurses per 1000 population and increased slower in the AGRs of the institutions, technicians and doctors per 1000 population. Notedly, some central and suburban districts even reversely experienced a negative average growth during this period. 
Fig.2 (a) and (b) illustrate the numbers of beds and nurses per 1000 population, respectively, across Shanghai's districts from 2010 to 2016. While the total amount of health resources was observed increasing across all the districts, there was a wide gap between central and suburban districts in the ratios and average growth rates of these two indicators. Compared with suburban areas, central ones had higher ratios of beds and nurses per 1000 population. From 2010 to 2016, we observed an increasing trend in the number of beds per 1000 population across mostly of districts, especially for main central district Huangpu, Jing'an, and Xuhui, rising from 15.10 to 17.52, 9.38 to 12.57 and 12.23 to 14.29 , respectively. Among these districts, Huangpu had the biggest average ratio in 2016, with above 17 beds per 1000 people. Jing'an and Xuhui were also above 10 in this ratio that year, higher than other districts, demonstrating higher bed density in the central areas of Shanghai. Reversely, that indicator of Chongming and Fengxian, which were located far from central areas of Shanghai, decreased noticeably from 4.94 to $4.52,4.20$ to 4.13 in the same year, respectively. A similar trend was also observed in the number of nurses per 1000 population during the period.

Figures 2 (c), (d) and (e) illustrate the numbers of institutions, technicians and doctors per 1000 population, respectively, across Shanghai's districts from 2010 to 2016. Though suburban districts occupied fewer average ratios of the institutions, technicians and doctors than urban ones, they were observed growing faster annually in these indicators during the period. For example, from 2010 to 2016, Fengxian, Chongming, Qingpu, and Jinshan were the fastest fourth of annually growth among other administrative divisions, with the AGRs of $22.39 \%, 21.15 \%$, $16.14 \%$ and $14.79 \%$, respectively. Aside from AGRs, each of these four suburban districts also had higher ratios of the institutions, technicians and doctors than other suburban districts, an even some central districts. For example, in 2016, Chongming district had 0.51 institutions per 1000 people, more than twice of the average number of institutions for central districts in the same year. It was also noticed that Huangpu reversely experienced a negative average growth rate of $-0.68 \%$, with decreasing from 0.33 in 2010 to 0.32 in 2016 . It was possibly due to this new district's administrative division merging in 2011, resulting in the decease of total number of health institutions. Similarly, both the number of technicians and doctors per 1,000 people showed the same trend as that observed for the number of institutions per 1,000 people. 
Inequality in health-resource allocation at the whole city level in Shanghai from 2010 to 2016

Table 4 and Fig. 3 present the Theil indices of health-resource allocation in Shanghai from 2010 to 2016. When vertically comparing data from the same year, the Theil indices of the technicians, doctors, nurses and beds were all greater than that for institutions. This indicated the existence of unbalance of workforce and bed allocation in Shanghai during this period. For example, in 2016, the Theil indices of the numbers of technicians, doctors, nurses and beds were $0.21,0.23,0.20$ and 0.18 , respectively, whereas the index of institutions was 0.06 . Similar unbalance also existed in other 6 years from 2010 to 2015.

Table 4 Theil indices related to health-resource allocation in Shanghai (2010-2016)

\begin{tabular}{lccccccc}
\hline \multicolumn{1}{c}{ Indicators } & 2010 & 2011 & 2012 & 2013 & 2014 & 2015 & 2016 \\
\hline Number of institutions & 0.09 & 0.08 & 0.08 & 0.06 & 0.06 & 0.06 & 0.06 \\
Number of beds & 0.19 & 0.19 & 0.19 & 0.19 & 0.19 & 0.19 & 0.18 \\
Number of technicians & 0.23 & 0.22 & 0.22 & 0.20 & 0.21 & 0.21 & 0.21 \\
Number of doctors & 0.19 & 0.19 & 0.19 & 0.17 & 0.18 & 0.18 & 0.20 \\
Number of nurses & 0.24 & 0.24 & 0.23 & 0.22 & 0.22 & 0.23 & 0.23 \\
\hline
\end{tabular}

As for Theil index trends for health-care resources in Shanghai from 2010 to 2016, the indices of all indicators exhibited a decline trend, except for the numbers of doctors. This indicated a reduction in the inequality with respect to most health-care resource indicators in Shanghai over the 7 years. For example, the Theil index for institutions decreased from 0.09 in 2010 to 0.06 in 2013, followed by a little increase to 0.06 in 2016 . The Theil index of beds also declined from 0.19 in 2010 to 0.18 in 2016 . The trend in Theil index for technicians and nurses were the same as that of institutions, with a decrease from 2010 to 2013 and then an increase until the period end. This range was $0.23-0.21$ for the technicians, and $0.24-0.23$ for the nurses, respectively. This indicated a reduction in the inequality of these two types of health-care workforce in that period.

However, for the Theil indices of the doctor, reverse trends were observed in the numbers of doctors during this period. For example, there was an overall increase trend over 7 years, from 0.19 in 2010 to 0.20 in 2016, which demonstrated that the problem of inequality of doctor allocation in Shanghai had not been solved. 


\section{Discussion}

This study analyzed the temporal trends and inequality of health-resource allocation at district level in Shanghai, noting trends of improvements in the quantity and inequality in healthresource allocation from 2010 to 2016. However, various regions were noticed to have unbalanced distributions of health workforce, especially for doctors, which exhibited serious inequalities in either number or temporal trend.

Firstly, this study observed that the number of institutions, the number of beds, the number of technicians, number of doctors, the number of nurses all increased over the 7 years. These results indicated that the Chinese government's goals of reforming the healthcare system to operate smoothly, and providing a safe, efficient, and convenient health service over past 7 years have been achieved. To expand and optimize health-care resources on the supply side, according to the "Healthy China 2030" planning outline and other health policy plans, China has integrated health subsystems by financially investing in health institutions to purchase and expand beds, equipment and other facilities; recruit and train technicians and doctors; make health institutions function reoriented; update the health-care service model based on the state of public health; and present a collaborative hierarchical medical system that meets people's health-care demands[1922]. This included not only perfecting plans for the geographical distribution of health-care resources across different regions and districts[23], but also maintaining a dynamic balance in allocation between facility and workforce. On the demand-side, the government has educated Chinese people about the "big health" concept to foster healthy lifestyles, as well as re-designed medical insurance to widen coverage among poorer people[24], under which an increasing number of patients were given reasonable access to health-care resources. Thus, the aforementioned measures of the Chinese and Shanghai governments have resulted in increased numbers of institutions, beds, technicians, doctors, and nurses across varied districts, and also reduced the inequality in health-resource allocation from 2010 to 2016. Numerous studies have reached similar results[25-28].

Secondly, this study observed regional differences in health-resource distribution at district level from 2010 to 2016. Health programs were unbalanced in their development when central and suburban districts were compared, which resulted in urban-rural regional disparity in healthcare resources. This was due to some historical reasons as followed. Most of public hospitals, especially tertiary hospitals were originally distributed in urban or municipal districts. They 
received more supports including fiscal reimbursement and policy inclination from Shanghai government than those located in rural districts. Furthermore, due to the new 2009 reform initiatives to strengthen the status of public hospital, plus the improved transportation convenience and accessibility to health care, the institutions located in urban districts were overwhelmed in term of beds, equipment and other facilities, forming a Matthew Effect that, the institutions in urban districts were visited by more and more people, while they were more financially invested by the government. Contrastedly, the institutions in rural areas were underdeveloped to form the socio-economic and health-care accessibility gaps between urban and suburban districts. Some relevant studies have also noted the distribution imbalance of institutions [26,27,29,30]. Furthermore, this effect also led to increasing numbers of the health workforce being attracted from suburban to central districts for pursuing higher salary, prospective career development in urban hospitals. We also found that some suburban districts grew faster than urban ones did in the numbers of institution, technician and doctor in light of AGRs. This was possibly attributed to some measures taken by Shanghai government to implement the strategy of " $5+3+1$ " since 2009 , which aimed to multiple the numbers of the tertiary hospitals and their workforce, equipment and other facilities in rural districts, in order to narrow the public hospital distribution gap between the urban and suburban districts and increase health care accessibility for residents of rural districts. For example, Chongming, Fengxian, and QingPu district were referred to the "3" sub-strategy targets of " $5+3+1$ " public hospital reform to update their public hospitals into tertiary hospitals to meet the demands of health-care in these three administrative divisions since 2009. This result on the regional difference is similar to those of studies that discovered an overcentralized health-care resource in urban areas and rapidly growing numbers of institution and workforce items in suburban areas in China[31-34]. Third, this study used the Theil index to analyze inequality in health-resource allocation. The index has some disadvantages, such as being complex to calculate and interpret; a wide variety when distribution varies regardless of the change that occurs in the top, middle, or bottom tier of resources; and the fact that when comparing populations with different sizes, the calculation is dependent on the number of individuals in the population or group. Nonetheless, this measurement method can still be robust when determining inequality within and between group components, with high sensitivity to the efficiency of health-resource allocation. This is because the index is decomposable by groups, can incorporate group-level data, and is particularly 
effective at paring effects in hierarchical data sets [35]. This study confirmed the inequality among technicians, doctors, nurses, beds and institutions from 2010 to 2016. On one hand, the Theil indices of workforce and beds were higher than that of institutions in Shanghai when comparing same year's data, demonstrating unbalanced distribution of health-care resources between institutions and workforce. This is attributable to the fact that, compared with expanding health institutions, recruiting and educating health workforce is a longer-term work to narrow the health-care resource distribution gap between urban and suburban districts. As mentioned above, technicians, doctors and nurses were more likely to work in bigger institutions in urban districts, given the relative advantages of salary and career development. Accordingly, for rural districts, it was harder to quickly attract or educate health workforce in a short time period based on condition of lower salary and socio-economic constrains, whereas expanding and opening branch institutions and facilities were easier to achieve. This result is consistent with the findings reported by Chen R. et.al[36], Liang D. et. al. [37], and Li D. et al. [38]. On the other hand, the Theil index of doctors, increased during this period, indicating worsening inequality in physician allocation. The possible reason could be that the elevated provision of human resources does not necessarily indicate a decline in inequity, as has been noted in other countries [39-42], or increasing numbers of rural doctors flow into larger urban tertiary institutions. Another reason is distribution disparity among different institutions. Perhaps Shanghai has developed a certain cultural tradition that people are more likely to choose to work in public sectors, such as government-affiliated sectors or public hospitals, resulting in the underdevelopment of private hospital sectors. Therefore, doctors with good quality had more probability of being employed in famous or public health institutions, while ones with not-good quality had to be in nonfamous or private health institutions, further exacerbating the disparities between doctors at different levels. This finding was similar to those of some relevant studies, which have confirmed the physician distribution gap among different regions or hospitals of various sizes[43-46].

The present study has several limitations. Firstly, the data used could potentially only reflect the health-resource allocation status in Shanghai at the cut-off because we could only obtain them from the Chinese Yearbooks, which are usually published officially at least 2 years after the year the data were collected. Therefore, crucial information could have been omitted from our data. In the future, a new study on changes in health-resource allocation from 2017 to the present, along with comparisons with the present study, can be conducted when the data are available. Secondly, 
this study did not consider the effect of the population's health outcomes on health-resource allocation. According to the health capacity paradigm theory [47], the population's health status in a region will have mutual effects on health-resource allocation in that area. Due to time and resource constraints, we did not consider these factors, which may have somehow affected the results. Thirdly, we selected indicators for health-resource allocation rather than indicators of the quality of health services. Factors represented by other unmeasured indicators may have influenced the results. Thus, integrating the indicators of health-resource allocation used in this study with those of health service quality may yield more robust results in a future study.

\section{Conclusion}

Health-care resources increased and inequality in resource allocation decreased in Shanghai from 2010 to 2016. This indicates the success of the measures taken by the Chinese government since its 2009 health care reform, specifically with respect to institutions, technicians, doctors in Shanghai. However, the distribution of health-care resources differed between urban and rural areas, and inequality of workforce, especially doctor, allocation had not been mitigated until 2016 since 2009's health care reform, demonstrating that the measures taken by the government to improve the attractivity of health institutions in rural areas and increase health care accessibility for residents there were needed to be strengthened further. Therefore, to achieve a regional balance in health-care resource distribution between central and rural areas and improve the equality of workforce allocation in Shanghai, policies should not pay attention to improve the socioeconomic levels in rural districts by raising income, make transportation conditions better, invest more fiscal funds and providing more access to health-care service for residents of rural districts. They should also focus on the balance of physician distribution between institutions at various levels, such as remobilization, job performance evaluation. To more deeply explore health-resource allocation, future studies will be conducted to integrate the indicators used in the present study with indicators of health service quality.

\section{Additional files}

Additional file 1: Changes for each indicator with whiskers box plot per every year from 2010 to 2016(per 1000). (PDF $179 \mathrm{~kb}$ )

\section{Abbreviations}

AGR: Annual Growth Rate.

\section{Declarations}


Ethics approval and consent to participate Not applicable

Consent for publication Not applicable

Availability of data and materials Data are available upon reasonable request.

Competing interests None declared

Funding This research were funded National social Science Foundation of China General Project (Grant No. 19BGL246); National social Science Foundation of China Major Project (Grant No. 18ZDA088); National social Science Foundation of China General Project (Grant No. 18BGL242); Key Program for Collaborative Innovation of Shanghai University of Medicine \& Health Science Grant No. SPCI-18-02-001). The funders had no role in the question design, analysis or interpretation.

\section{Authors' contribution}

ED, HW, TW and LZ designed the study together, acquired the data and developed the statistical plan. MC and TX carried out the survey. HW performed the statistical analysis. ED, XS,TW and LZ interpreted the analysis. ED, XS, LZ and TW drafted and revised the manuscript. All authors read and approved the final manuscript.

\section{Author details}

1 School of Nursing and Health Management, Shanghai University of medicine \& Health Science, 279 Zhouzhu Road, Pudong new District, Shanghai 201318, Shanghai, China;2 School of Media and Communication, Shanghai Jiao Tong University, Shanghai 200240, Shanghai, China;3 Shanghai Tenth People's Hospital affiliated to Tongji University, Shanghai, China;4 Tongji University School of Medicine, Shanghai, China; 5 School of International and Public Affairs, Shanghai Jiao Tong University.1954 Huashan Road, Xuhui District, Shanghai 200030, Shanghai, China;6 Department of Outpatient and Emergency, Renji Hospital Affiliated to Shanghai Jiao Tong University School of Medicine, NO.160 Pujian Road, Pudong new District, Shanghai,200127,China; 7 Department of Health Services Research \& Administration, College of Public Health, 984350 University Nebraska Medical Center, Omaha, NE 68198-4350;8 College of Arts and Media, Tongji University, Shanghai 200092China.

\section{References}

1. i Casasnovas GL, Rivera B, Currais L, editors. Health and economic growth:findings and policy implications. Mit Press,2005. 
2. Cutler DM, Lleras-Muney A, Vogl T. Socioeconomic status and health:dimensions and mechanisms. National Bureau of Economic Research 2008 ;Sep10.

3. Culyer AJ, Wagstaff A. Equity and equality in health and health care. Journal

of health economics 1993;12(4):431-57.

4.Tao Y, Henry K, Zou Q, et al. Methods for measuring horizontal equity in health resource allocation: a comparative study. Heal Econ Rev 2014;4:1-10.

5.Szalai J. Inequalities in access to health care in Hungary. Social Science \& Medicine 1986; 22:135-40.

6. Chen LY, Chen SY. "1560" medical circle has basically built in Shanghai, and at least one tertiary hospital is available in each

district.http://district.ce.cn/newarea/roll/201212/28/t20121228_23983925.shtml ( accessed 28 Dec. 2012).

7. Cheng T M. Explaining Shanghai's health care reforms, successes, and challenges. Health Affairs 2013;32(12): 2199-2204.

8. Bao Y, Du XL, Zou LM, et. al. Analysis and policy suggestion of hospitalizing intension of residents in Shanghai. Journal of Shanghai Jiaotong University (Medical Science) 2010;30(08):948-950+955(in Chinese).

9.Wang S, Xu J, Jiang X, et al. Trends in health resource disparities in primary health care institutions in Liaoning Province in Northeast China. International journal for equity in health 2018;17:1-8.

10. Sun J. Equality in the distribution of health material and human resources in Guangxi: evidence from Southern China. BMC research notes 2017;10:429.

11. Sun J, Luo H. Evaluation on equality and efficiency of health resources allocation and health services utilization in China. International journal for equity in health 2017;16:127.

12 Chen R, Zhao Y, Du J, Wu T, Huang Y, Guo A. Health workforce equity in urban community health service of China. PLoS One 2014;9:e115988.

13. Pan J, Shallcross D. Geographic distribution of hospital beds throughout China: a countylevel econometric analysis. International journal for equity in health 2016;15:179.

14. Zhang Y, Wang Q, Jiang T, Wang J. Equity and efficiency of primary health care resource allocation in mainland China. International journal for equity in health 2018;17:140. 
15. FAN Xin , ZHANG Dingyue, LI Xu, et al. Distribution equality of mental health facilities and psychiatric beds in Shanghai based on Theil index. Chinese Mental Health Journal 2018; 32):829-34(in Chinese).

16.Theil H. Economic and information theory North Holland Publish. Co.,Amsterdam,1967.

17.StataCorp 15. Stata survey data reference manual, release 12. College Station: StataCorp LP; 2014.

18.ESRI. ArcGIS Desktop: Release 10. Environmental Systems Research Institute Redlands. California; 2011.

19. KUANG Li. Strategy for optimizing the mechanism of healthcare competition: Establishing the vertical integrated healthcare delivery systems. Chinese Journal of Health Policy 2012;5:349(in Chinese).

20. Zhao Dan-dan. The reality and preliminary analysis on medical resource and their vertical integration in Shanghai. Chinese Health Resources 2008;11:259-62(in Chinese).

21. Iriart C, Merhy EE, Waitzkin H. Managed care in Latin America: the new common sense in health policy reform. Social Science \& Medicine 2001;52:1243-53.

22. Barr DA: Introduction to US Health Policy: the organization, financing, and delivery of health care in America: JHU Press; 2016.

23.Ren Wenjie. The Path Selection of Medical Resource Optimizing Allocation. Chinese Health Resources 2014; 27:23-5(in Chinese).

24. Hai QS, Jin YJ. Connotation and Basic Characteristics of Great Health Concept. Journal of Traditional Chinese Medicine 2017;58(13):1085-1088(in Chinese).

25. Zhao Dan-dan. the realty and preliminary analysis on medical resource and their vertical integration in Shanghai. Chinese Health Resources 2008;11:259-62(in Chinese).

26.Zhang LF,Li LQ. Study on the Equilibrium of Spatial Allocation of Medical Resources at Different Levels in Shanghai. Urban Development Studies 2019;26(06):1-6(in Chinese).

27.Zhang LF. Study on the Equilibrium and Optimization of Medical Resource Allocation in Metropolitan Areas: A Case Study of Shanghai. Nanjing Journal of Social Sciences 2019;(02):65-72(in Chinese).

28.Wang D, Ma L, Gong EJ et al. Analysis of the Dominant Diseases of Tertiary Public Hospitals Newly Built in Suburbs in Shanghai. Chinese Hospital Management 2016;36(06):1921(in Chinese). 
29. Shen WW, Bao Y. Analysis of nursing service needs of old people in Shanghai nursing institutions for the aged and policy recommendations. Journal of Shanghai Jiaotong University (Medical Science) 2015; 35(4):581-584.

30.Shi $\mathrm{J}$ et al. Disparities in mental health care utilization among inpatients in various types of health institutions: a cross-sectional study based on EHR data in Shanghai, China. BMC public health, 2019, 19(1):1023.

31.Dong EH, Li GH, Cai YY, et al. A review on regional difference in healthcare resource allocation. Chinese Health Resource 2016;19(05):390-393(in Chinese).

32.Fan XA. The lag of medical reform affects the effectiveness of medical insurance, and it is the key for doctors to go to the grassroots level. China Health Insurance 2014;(12):23-24(in Chinese).

33.Gong SW, Li ZG, Xu Y, et al. Research on regional distribution differences of healthcare resources in China from a perspective of accessibility. Chinese Journal of Hospital Administration 2011;(05):325-330(in Chinese).

34. Xu Z, Geng H, Zeng Z. The analysis of medical resources allocation between Nanjing thirdlevel hospitals and community health center. Chinese Health Service Management 2012;(05):335-338(in Chinese).

35.Tao Y, Henry K, Zou Q, Zhong X. Methods for measuring horizontal equity in health resource allocation: a comparative study. Health economics review 2014;Dec;4:10.

36. Chen $\mathrm{R}$ et al. Health workforce equity in urban community health service of China. PLoS One2014; 9(12):e115988.

37. Liang D et al. Does rapid and sustained economic growth lead to convergence in health resources: The case of China from 1980 to 2010. Inquiry (United States) 2016;53(1).

38. Li D et al. Unequal distribution of health human resource in mainland China: What are the determinants from a comprehensive perspective? International Journal for Equity in Health 2018;17(1).

39. Paraje G, Vásquez F. Health equity in an unequal country: the use of medical services in Chile. International journal for equity in health 2012;11:81.

40. Glorioso V, Subramanian SV. Equity in access to health care services in Italy. Health services research 2014;49:950-70. 
41. Smith S, Normand C. Equity in health care: the Irish perspective. Health Economics, Policy and Law 2011;6:205-17.

42. Ghosh S. Equity in the utilization of healthcare services in India: evidence from National Sample Survey. International journal of health policy and management 2014;2:29

43.Zou YJ. Comments on brain drain in private hospitals of China. Jiangsu Healthcare Administration 2010;21(01):46-47(in Chinese).

44. Zhang XJ, Zhu K. Equity in the Distribution of Human Resources for Health in China during 2004-2015. Chinese General Practice 2018;21(1):82-87.

45.Anand $\mathrm{S}$ et al. China's human resources for health: quantity, quality, and distribution. The Lancet 2008;372(9651):1774-1781.

46.Zhu B et al. Detecting the priority areas for health workforce allocation with LISA functions: An empirical analysis for China. BMC Health Services Research 2018;18(1).

47.Chakraborty R, Chakraborti C. India, health inequities, and a fair healthcare provision: A perspective from health capability. Journal of Human Development and Capabilities 2015;16:567-80. 


\section{Figures}
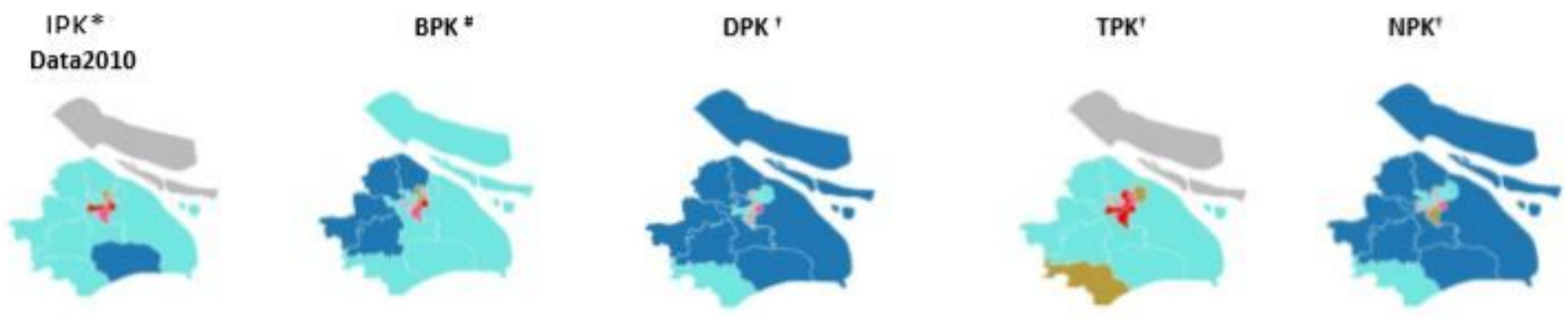

\section{Data2013}
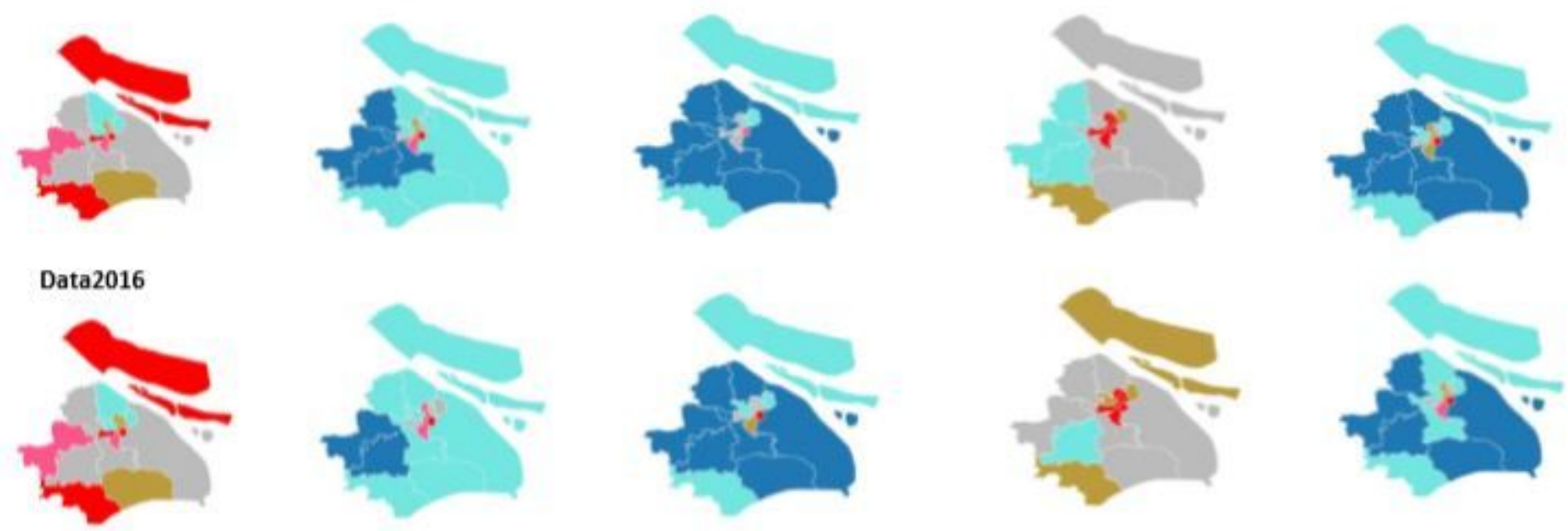

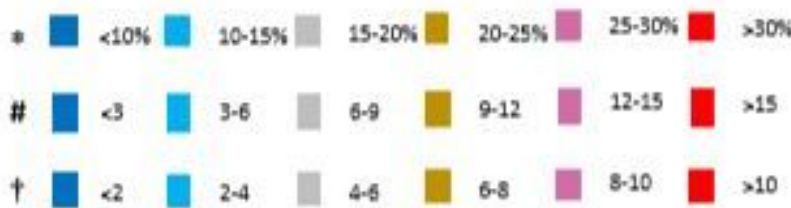

Fig. 1 Time Trends and Geographic Distribution in the Health-care Resource Allocation Across all Shanghai's Districts From 2010 to 2016.IPK: Number of institutions per 1000 people ;BPK: Number of beds per 1000 people; DPK: Number of doctors per 1000 people; TPK: Number of technicians per 1000 people; NPK: Number of nurses per 1000 people.

\section{Figure 1}

Time Trends and Geographic Distribution in the Health-care Resource Allocation Across all Shanghai's Districts From 2010 to 2016.IPK: Number of institutions per 1000 people ;BPK: Number of beds per 1000 people; DPK: Number of doctors per 1000 people; TPK: Number of technicians per 1000 people; NPK: Number of nurses per 1000 people. 
(a)

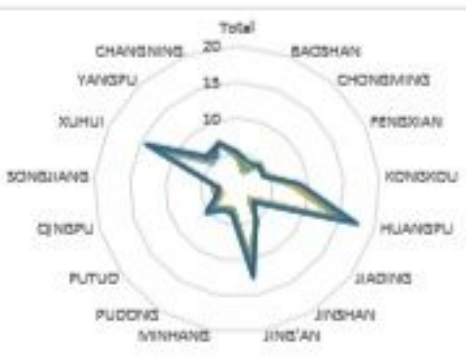

(b)

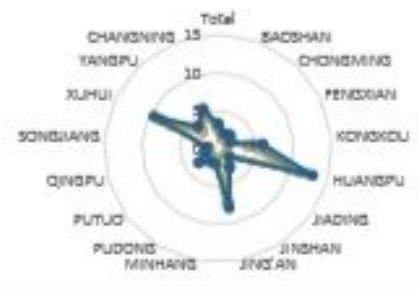

(c)

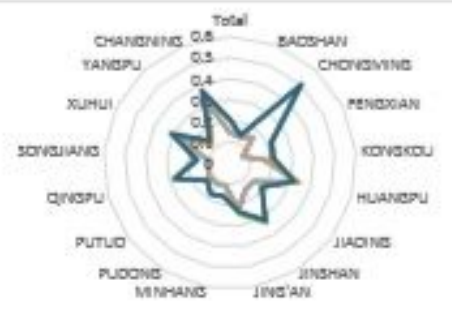

(d)

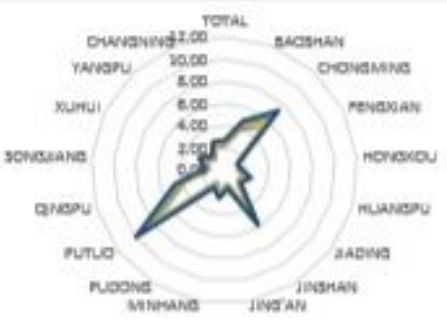

(e)

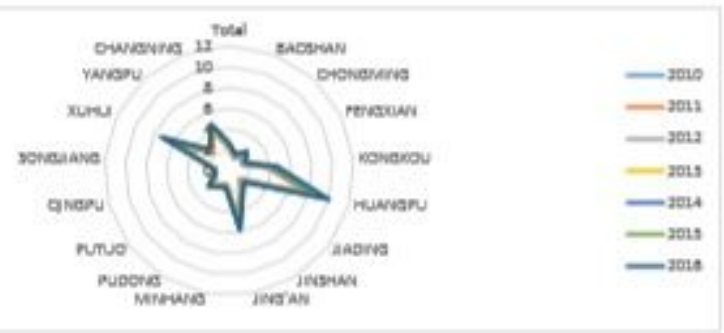

\section{Figure 2}

Per 1000 Health Resources Across the Districts in Shanghai From 2010 to 2016 (a) presents per 1000 beds across the districts in Shanghai from 2010 to 2016; (b) presents per 1000 nurses across the districts in Shanghai from 2010 to 2016 ; (c) presents per 1000 health institutions across the districts in Shanghai from 2010 to 2016; (d) presents per 1000 technicians across the districts in Shanghai from 2010 to 2016; (e) presents per 1000 doctors across the districts in Shanghai from 2010 to 2016;

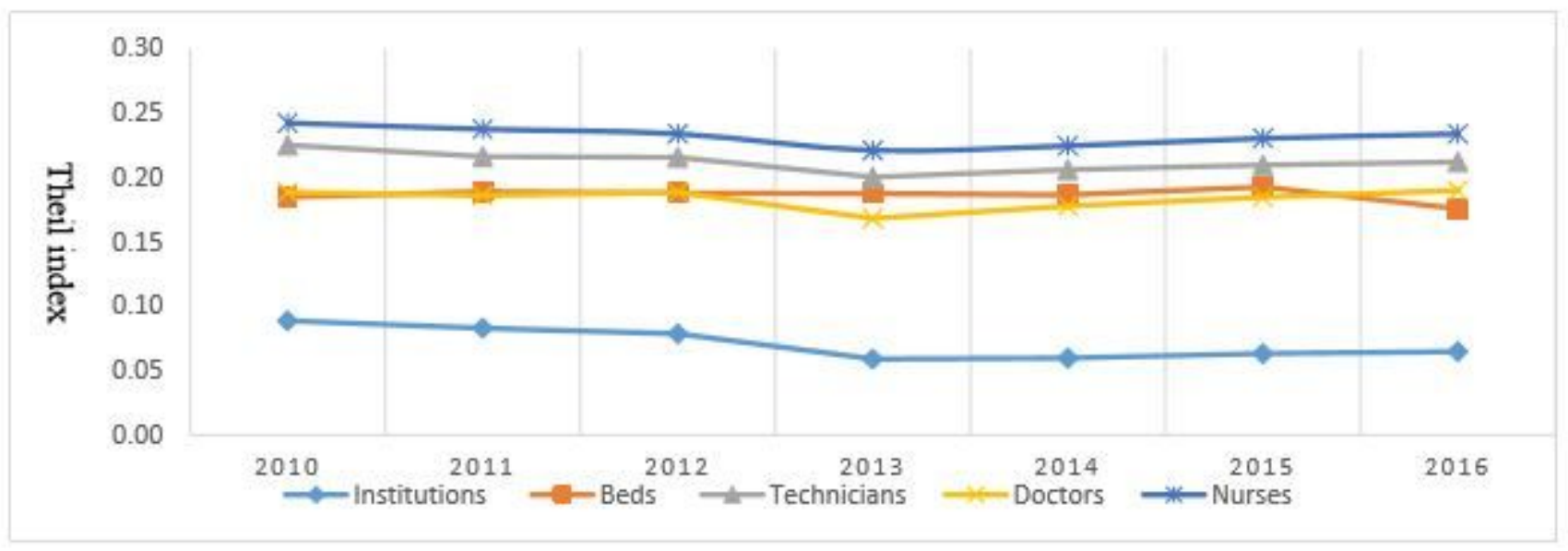

Figure 3 
Trends of The Theil Indexes For the Health Resources in Shanghai From 2010 to 2016

\section{Supplementary Files}

This is a list of supplementary files associated with this preprint. Click to download.

- additionalfile.pdf 